\title{
MASSIVE PULMONARY EMBOLISM IN A BOY AGED 9
}

\author{
BY \\ MAUREEN CASHMAN \\ From the Royal Berkshire Hospital, Reading \\ (RECEIVED FOR PUBLICATION JULY 9, 1956)
}

Massive pulmonary embolism is not normally a post-operative complication to be feared in a boy of 9 and no description of another case in childhood has been found in the literature: it must therefore be a rare event.

\section{Case History}

The patient, a boy aged 9, was admitted to the children's ward at the Royal Berkshire Hospital in March, 1956, as a case of suspected acute appendicitis. Three days earlier he had complained of feeling sick and giddy and also had some central abdominal pain. He vomited several times that day and during the next two days and the pain persisted. The day before admission he passed several loose stools and the night before admission had been feverish. He had had a head cold with a little cough 10 days earlier which had not cleared up completely.

On admission he was a boy of good physique who was flushed but did not look ill. He was febrile (temperature $102^{*} \mathrm{~F}$., pulse 110), the tongue was furred and the pharynx and tonsils were injected. The chest was clinically clear and heart sounds were normal. There was lower abdominal tenderness, mainly on the right with muscle guarding. Bowel sounds were present. There was some tenderness per rectum to the right. Urine was normal.

The lower abdominal tenderness and guarding increased during three hours' observation and appendicectomy was decided upon. At operation Mr. G. L. Bohn found a high paracolic appendix, the tip reaching

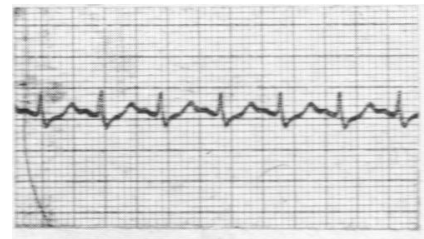

1

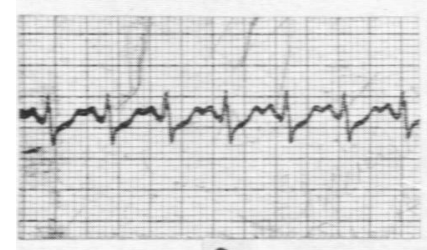

2

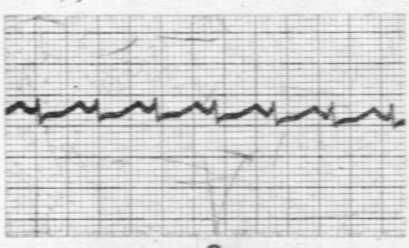

3

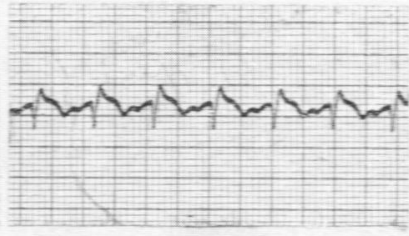

aVR

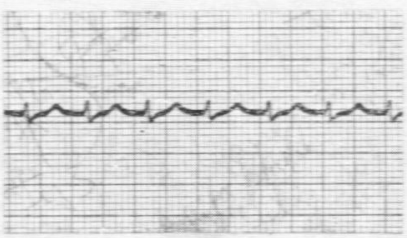

aVL

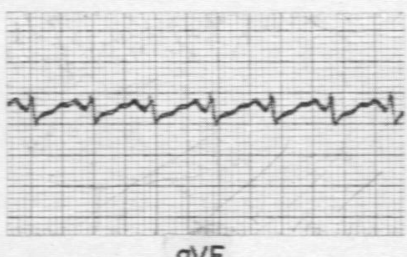

QVF

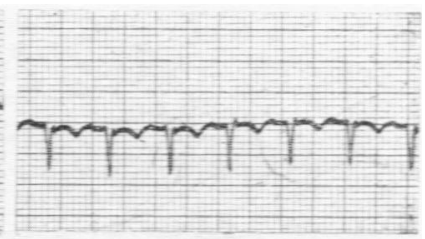

VI

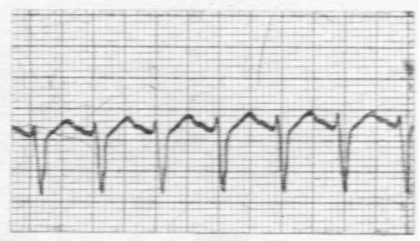

V3

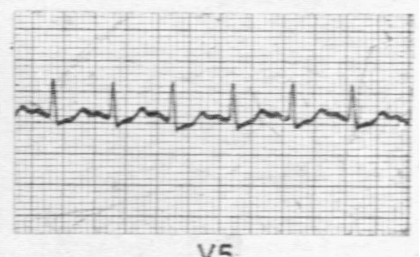

V5

Fig. 1.-Electrocardiograph four hours after embolism. Prominent S.1, tachycardia. 


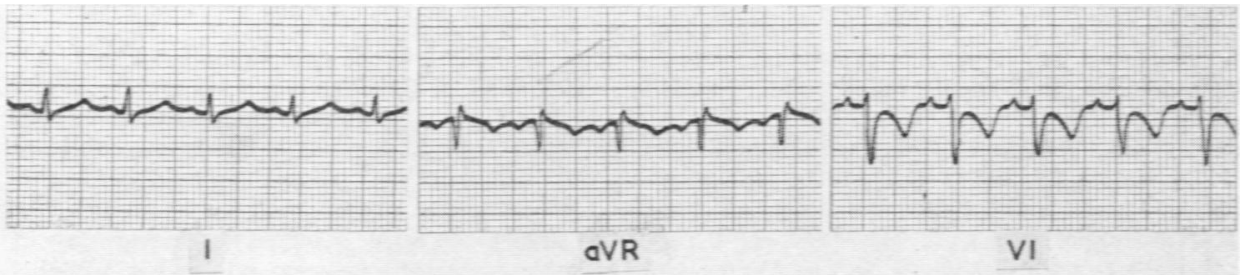

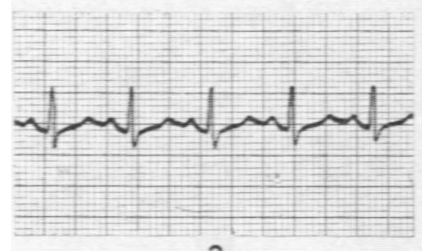

2

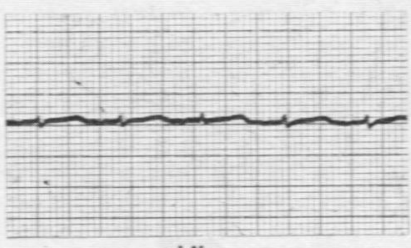

aVL

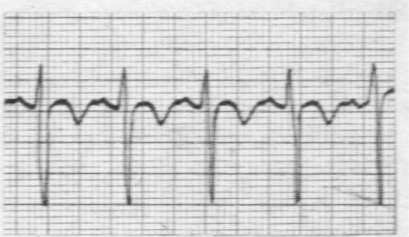

V3

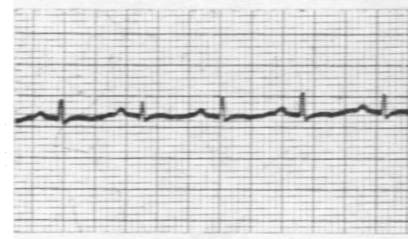

3

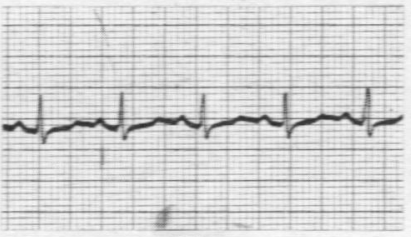

aVF

Fyg. 2-Electrocardiograph four days after embolism. Steep inversion of T.V.1 and T.V.3, with displacement of transition zone to the left.

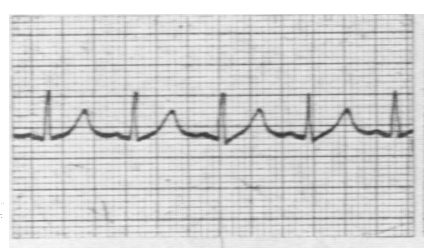

1

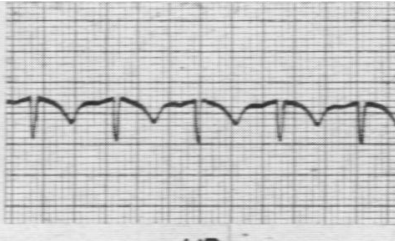

aVR

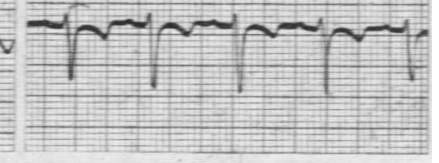

VI

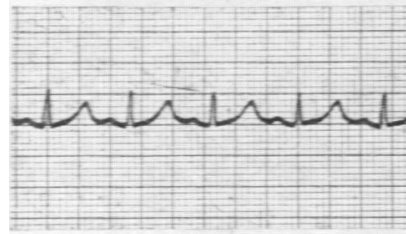

2

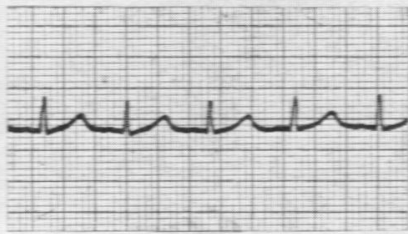

aVL

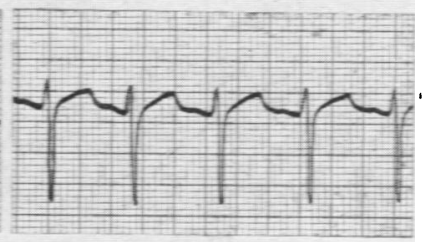

V3

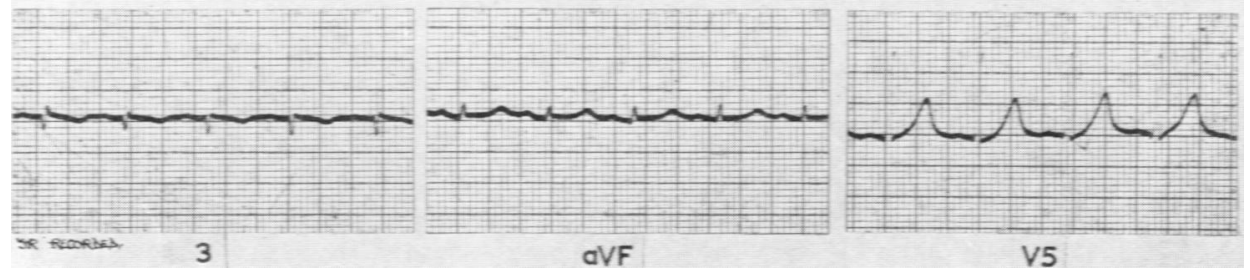

FiG. 3.-Electrocardiograph 26 days after embolism. Return to normal. 
to the liver. It was difficult to remove but was not inflamed.

No chemotherapy was given and over the next four days the temperature and upper respiratory tract infection settled. A chest radiograph was clear. Culture from stools grew no pathogens.

On the fifth post-operative day he sat up for tea and on the following day he got up again in the afternoon. He had been sitting in a chair for a few minutes when he complained of sudden severe pain in the left side of the chest and became dyspnoeic, pale and sweating. The degree of collapse rapidly became very severe.

He was put in an oxygen tent and given 10 minims of nepenthe and afterwards there was some improvement but a state of severe shock, with a hardly perceptible wrist pulse, persisted for about six hours. There was some blood-tinged sputum.

The first electrocardiogram taken four hours after the onset of symptoms showed a tachycardia and a prominent $\mathrm{S}$ in lead 1 (Fig. 1).

As the clinical picture was that of pulmonary embolism as seen in the adult, he was given 10,000 units of heparin intravenously eight hourly for three doses. An initial dose of $100 \mathrm{mg}$. of 'dindevan' was followed by $75 \mathrm{mg}$. the next day, then $25 \mathrm{mg}$. twice daily, the dose thereafter depending on prothrombin estimation. 'Dindevan' was continued for three weeks, maintaining the prothrombin efficiency at 35 to $50^{\circ}$. He was given intramuscular penicillin in addition for the first five days.

He remained extremely ill for $\mathbf{4 8}$ hours and then made a steady recovery. There was never any calf or leg tenderness and there were no further embolic episodes. No physical signs appeared in the chest.

An E.C.G. repeated four days after the onset showed a steep inversion of T.V.1 and T.V. 3 with displacement of the transition zone to the left (Fig. 2) and this was still present five days later. After a further week the tracing was within normal limits (Fig. 3).

The E.C.G. appearances of transient acute cor pulmonale are characteristically those of massive pulmonary embolism and are thought to be due to sudden clockwise rotation of the heart.

Since discharge from hospital one month after the illness the patient has been well and is now back at school and completely active. A chest film is clear and the heart shadow normal.

A case of this sort immediately involves a number of the hospital team and I am grateful to Dr. J. J. Kempton, Mr. G. L. Bohn, Dr. A. Anderson and Dr. A. L. Tarnoky for guidance and for much advice and help. 